\title{
Cardiac effects of non-cardiac drugs
}

\author{
Ingrid Hopper, Clinical pharmacology fellow, Alfred Health, Melbourne
}

\section{Summary \\ Drugs prescribed for non-cardiac conditions can have unexpected and serious cardiac effects. These may occur while taking the drug or can be delayed for years. Often these adverse effects are not recognised until the postmarketing phase of drug development. An underlying cardiac abnormality can be a predisposing factor. Drugs with cardiac adverse effects include clozapine, rosiglitazone, non-steroidal anti-inflammatory drugs, tumour necrosis factor inhibitors, cancer chemotherapy and drugs for Parkinson's disease.}

Key words: arrhythmia, heart failure, myocardial infarction.

(Aust Prescr 2011;34:52-4)

\section{Introduction}

Many drugs have unexpected cardiovascular effects. Health professionals should be aware of the potential for non-cardiac drugs to have effects on the heart when prescribing to patients with or without cardiac disease or cardiac risk factors. Data on the cardiac effects of non-cardiac drugs can be difficult to interpret. Adverse effects may become apparent late in the development of the drug, such as during review of the safety data or in the postmarketing phase. Trials may not have specified cardiovascular outcomes as an end point and the incidence of cardiac events can be low, so evidence is often derived from post hoc analyses or meta-analyses.

\section{Myocardial infarction}

Patients and physicians may be unaware that even some over-thecounter medicines may increase the risk of myocardial infarction.

\section{Non-steroidal anti-inflammatory drugs}

Non-steroidal anti-inflammatory drugs (NSAIDs) are widely used analgesics. Clinical trials of COX-2 inhibitors showed a slight increase in the rate of serious thrombotic vascular events, mostly myocardial infarction, compared with placebo., ${ }^{1,2}$ Rofecoxib has been withdrawn from the market. Studies with celecoxib are conflicting but an increased risk of myocardial infarction probably exists with sustained use of high doses, especially in patients who already have a high cardiovascular risk. ${ }^{3}$

The cardiovascular problems observed with COX-2 inhibitors prompted further study of the older non-selective NSAIDs. Various observational studies and meta-analyses found that diclofenac, and possibly ibuprofen, were associated with an increased risk of myocardial infarction. ${ }^{4,5}$ This relationship is dose-dependent, with higher doses resulting in more events. Evidence of the adverse effect of NSAIDs in patients with cardiovascular disease is scant, but a higher cardiovascular risk may potentiate the drugs' cardiotoxicity. NSAIDs, including COX-2 inhibitors, should therefore be used for the shortest time possible at the lowest effective dose.

\section{Thiazolidinediones}

The 'glitazones' are used as add-on treatment for patients with type 2 diabetes. Rosiglitazone was associated with increased rates of myocardial infarction, but not cardiovascular mortality, in a recently updated meta-analysis of 56 randomised controlled trials involving 35531 patients. ${ }^{6}$ The US Food and Drug Administration estimated that during 1999-2006 an excess 41000 to 205000 major cardiovascular events were potentially attributable to rosiglitazone. ${ }^{6}$ Rosiglitazone has been removed from the European market and access is restricted in Australia and the US. Pioglitazone appears to be a safer alternative.

\section{Antiretrovirals}

Highly active antiretroviral therapy has been associated with significant improvements in long-term prognosis in HIV infection. However, it can result in adverse metabolic changes which increase the risk of diabetes and cardiovascular disease, including increases in cholesterol and triglycerides, insulin resistance and abdominal adiposity. The use of protease inhibitors in particular has been associated with an increase in myocardial infarction. ${ }^{7}$ The relative contribution of the medications, lifestyle and the effect of HIV itself on cardiovascular disease is difficult to elucidate.

\section{'Triptans'}

The $5 \mathrm{HT}_{1}$ agonists used in migraine cause vasoconstriction of the cerebral vessels. $5 \mathrm{HT}_{1}$ receptors also exist in lower numbers and to a lesser extent in coronary arteries. There are rare reports of myocardial infarction, as well as other cardiovascular events, usually in patients with unrecognised cardiovascular disease, although some have occurred in patients with no cardiovascular disease and were thought to be due to vasospasm. ${ }^{8}$ Cardiac risk stratification should therefore be undertaken before patients are prescribed these drugs.

\section{Heart failure}

Heart failure occurs as a complication of a variety of drugs. Patients with identifiable cardiac risk factors such as previous myocardial infarction, hypertension and advanced age are 
at greater risk. Other risk factors relate to any underlying cardiovascular disease.

\section{Non-steroidal anti-inflammatory drugs}

NSAIDs do not appear to cause new occurrence of heart failure, however their use is strongly correlated with relapse of heart failure. ${ }^{9}$ Patients known to have heart failure should therefore avoid NSAIDs. The risks are dose-dependent. Diclofenac appears to be associated with the greatest risk, but heart failure has been observed with all NSAIDs including COX-2 inhibitors.

\section{Thiazolidinediones}

The glitazones are thought to cause fluid retention by increasing sodium reabsorption in the distal nephron. This causes peripheral oedema and can worsen existing heart failure or cause new onset heart failure. Randomised controlled trials have clearly shown increased rates of hospitalisation for heart failure, but not increased mortality. However, these trials have generally excluded patients with symptoms of New York Heart Association (NYHA) class III and IV heart failure. ${ }^{6}$ These drugs are therefore contraindicated in heart failure.

\section{Cancer chemotherapy}

The risk of cardiac complications associated with cancer treatment can be as great as the risk of recurrence of the cancer, particularly if there has been chest wall irradiation. ${ }^{10}$ Anthracyclines (doxorubicin and daunorubicin), used in haematological and solid organ tumours, cause significant cardiac toxicity. Heart failure, typically a dilated or restrictive cardiomyopathy, develops within one month to a year after treatment, although it can occur after a decade or more. The incidence is directly related to the cumulative dose. At the recommended maximum lifetime dose of doxorubicin $\left(550 \mathrm{mg} / \mathrm{m}^{2}\right)$, heart failure is observed in $7 \%$ of patients and increases rapidly above this maximum, although it can also occur with smaller doses. The patient's cardiac function should be monitored for life. Cyclophosphamide has also been shown to cause heart failure.

Trastuzumab is a recombinant IgG monoclonal antibody used in the treatment of human epidermal growth receptor-2 (HER-2) positive breast cancers. When used as monotherapy, trastuzumab is associated with a $3-7 \%$ increase in cardiac events, of which $2-4 \%$ are NYHA class III/IV heart failure. This incidence increases to as high as $27 \%$ when used in combination with anthracyclines. There is no clear relation to dose, and heart failure is often reversible, with re-initiation of therapy well tolerated.

Tyrosine kinase inhibitors, such as sunitinib, are small molecules which inhibit tumour growth and angiogenesis in metastatic renal cell carcinoma and certain gastrointestinal tumours. Heart failure and asymptomatic reduction in left ventricular ejection fraction has been observed in $10-15 \%$ of patients. Most cases improved when the drug was stopped.

\section{Tumour necrosis factor inhibitors}

The biological therapies that inhibit tumour necrosis factor are used to treat immune mediated inflammatory disease. These drugs were initially trialled for the treatment of heart failure, but in fact worsened the condition, and also caused heart failure in patients without any predisposing factors. Symptoms are mostly reversible when therapy is stopped, but deaths have occurred. ${ }^{11}$

\section{Complementary therapies}

Use of complementary therapies is common, especially in patients with chronic diseases, including heart failure. Evidence regarding their cardiac effects is scant and mostly anecdotal. ${ }^{12}$ Herbal therapies have the potential to interact with drugs with narrow therapeutic index, including digoxin and warfarin. They can also reduce or potentiate pharmacological effects of cardiovascular medications. ${ }^{13}$ Liquorice can cause fluid retention and precipitate heart failure, as well as hypertension.

\section{Myocarditis}

Inflammation of the myocardium can be caused by the direct effect of drugs or through an immune mechanism.

\section{Clozapine}

Myocarditis has been observed to occur at a rate of $0.7-1.2 \%$ in users of the antipsychotic, clozapine. This is 10000 times the background rate in the general population. ${ }^{14}$ Clozapineinduced myocarditis occurs in the first eight weeks of therapy. It is an unpredictable idiosyncratic reaction, unrelated to dose. Symptoms range from the mild to cardiogenic shock and death. Monitoring of troponin is recommended when starting clozapine. Echocardiography is used to assist in diagnosis, assess severity and follow recovery. Clozapine-induced myocarditis is mostly reversible with early recognition, so withdrawal of the drug is the mainstay of treatment along with supportive therapy.

Cardiomyopathy with longer term dysfunction of cardiac myocytes is also seen with clozapine therapy at a rate of $0.1 \%$. It can occur at any time during therapy but usually after the first eight weeks. It is not clear whether this is due to direct cardiotoxic effects of the drug, or evolution of unrecognised myocarditis.

\section{Valvular heart disease}

The drugs which have been shown to cause valvular heart disease include ergot alkaloids used in migraine prophylaxis and the now withdrawn appetite suppressant fenfluramine. ${ }^{15}$ The ergot-derived dopamine agonists cabergoline and pergolide, used in Parkinson's disease, have been associated with fibrotic reactions in the heart valves with consequent valvular regurgitation. Rates of clinically important regurgitation in the order of $25 \%$ have been found when these drugs are used for more than a year in Parkinson's disease. ${ }^{16}$ The severity of the regurgitation is related to dose and duration of therapy. Regression of the valvular lesion has generally been observed after treatment stops. 


\section{Arrhythmia}

Many drugs can prolong the QT interval on the ECG. ${ }^{17}$ This can cause life-threatening ventricular tachycardias including torsades de pointes. It is the leading cardiac reason for which drugs are withdrawn from the market, and as a consequence measurement of the QT interval has become an important part of drug development.

Prolongation of the QT interval has been associated with tricyclic antidepressants, antipsychotics, stimulant drugs such as amphetamines, as well as erythromycin and other macrolides, especially in the presence of drugs which affect metabolism such as verapamil, diltiazem and ketoconazole. ${ }^{18}$

It is difficult to identify which drugs may cause atrial fibrillation because of its high background rate in the community. High-dose corticosteroids have been observed to cause this arrhythmia. Bisphosphonates are associated with a small increased risk, as is the cholinesterase inhibitor donepezil used in Alzheimer's disease. ${ }^{19}$

Bradycardia can be caused by cholinergic drugs such as neostigmine and clonidine. Sinus tachycardia can be caused by adrenergic agonists, such as beta agonists, and antidepressants including duloxetine and venlafaxine.

\section{Conclusion}

Various non-cardiac drugs can have off-target effects on the heart that may not be expected based on knowledge of the pharmacology. A thorough medication history should be sought from all patients, including drugs taken in the recent and distant past. The use of certain drugs, in particular anthracyclines, mandates lifelong cardiac follow-up, and both patients and prescribers should understand this. The cardiac risk profile and relevant comorbidities should be considered before prescribing non-cardiac drugs which have a potential for cardiac adverse effects.

\section{Acknowledgement}

Professor Henry Krum and Ms Julie Lord assisted in the preparation of this manuscript.

\section{References}

1. Bresalier R, Sandler RS, Quan H, Bolognese JA, Oxenius B, Horgan K, et al; Adenomatous Polyp Prevention on Vioxx (APPROVe) Trial Investigators. Cardiovascular events associated with rofecoxib in colorectal adenoma chemoprevention trial. N Engl J Med 2005;352:1092-102.

2. Solomon SD, McMurray JJ, Pfeffer MA, Wittes J, Fowler R, Finn P, et al; Adenoma Prevention with Celecoxib (APC) Study Investigators. Cardiovascular risk associated with celecoxib in a clinical trial for colorectal adenoma prevention. N Engl J Med 2005;352:1071-80.

3. Solomon SD, Wittes J, Finn PV, Fowler R, Viner J, Bertagnolli MM et al; Cross Trial Safety Assessment Group. Cardiovascular risk of celecoxib in 6 randomized placebocontrolled trials: the cross trial safety analysis. Circulation 2008;117:2104-13.
4. McGettigan P, Henry D. Cardiovascular risk and inhibition of cyclooxygenase: a systematic review of the observational studies of selective and nonselective inhibitors of cyclooxygenase 2. JAMA 2006;296:1633-44.

5. Kearney P, Baigent C, Godwin J, Halls H, Emberson J, Patrono $C$. Do selective cyclo-oxygenase-2 inhibitors and traditional non-steroidal anti-inflammatory drugs increase the risk of atherothrombosis? Meta-analysis of randomised trials. BMJ 2006;332:1302-8.

6. Nissen SE, Wolski K. Rosiglitazone revisited. An updated meta-analysis of risk for myocardial infarction and cardiovascular mortality. Arch Intern Med 2010;170:1191-201.

7. Mondy K, Tebas P. Cardiovascular risks of antiretroviral therapies. Annu Rev Med 2007;58:141-55.

8. Martin VT, Goldstein JA. Evaluating the safety and tolerability profile of acute treatments for migraine. Am J Med 2005;118 Suppl 1:S36-44.

9. Feenstra J, Heerdink ER, Grobbee DE, Stricker BH. Association of nonsteroidal anti-inflammatory drugs with first occurrence of heart failure and with relapsing heart failure: the Rotterdam Study. Arch Intern Med 2002;162:265-70.

10. Minami M, Matsumoto S, Horiuchi H. Cardiovascular sideeffects of modern cancer therapy. Circ J 2010;74:1779-86.

11. Setoguchi S, Schneeweiss S, Avorn J, Katz JN, Weinblatt ME, Levin $\mathrm{R}$, et al. Tumour necrosis factor-alpha antagonist use and heart failure in elderly patients with rheumatoid arthritis. Am Heart J 2008;156:336-41.

12. Ernst E. Cardiovascular adverse effects of herbal medicines: a systematic review of the recent literature. Can J Cardiol 2003;19:818-27.

13. Tachjian A, Maria $V$, Jahangir A. Use of herbal products and potential interactions in patients with cardiovascular diseases. J Am Coll Cardiol 2010;55:515-25.

14. Layland JJ, Liew D, Prior DL. Clozapine-induced cardiotoxicity: a clinical update. Med J Aust 2009;190:190-2.

15. Bhattacharyya S, Schapira AH, Mikhailidis DP, Davar J. Druginduced fibrotic valvular heart disease. Lancet 2009;374:577-85.

16. Zanettini R, Antonini A, Gatto G, Gentile R, Tesei S, Pezzoli G. Valvular heart disease and the use of dopamine agonists for Parkinson's disease. N Engl J Med 2007;356:39-46.

17. Jayasinghe R, Kovoor P. Drugs and the QTc interval. Aust Prescr 2002;25:63-5.

18. Roden D. Drug-induced prolongation of the QT interval. N Engl J Med 2004;350:1013-22.

19. Van der Hooft CS, Heeringa J, van Herpen G, Kors JA, Kingma JH, Stricker BH. Drug induced atrial fibrillation. J Am Coll Cardiol 2004;44:2117-24.

Conflict of interest: none declared

\section{Self-test questions}

The following statements are either true or false (answers on page 59)

3. Non-steroidal anti-inflammatory drugs should not be used if a patient has a history of heart failure.

4. The $5 \mathrm{HT}_{1}$ agonists used to treat migraine may cause coronary vasodilation. 